\title{
Efficacité comparée de trois méthodes de diffusion d'informations rizicoles par des vidéos au Sud du Bénin
}

\author{
Thomas Davito ${ }^{1}$, Florent Okry ${ }^{2, *}$, Augustin Kouevi ${ }^{1}$ et Simplice Vodouhe ${ }^{1}$ \\ ${ }^{1}$ Faculté des Sciences Agronomiques (FSA), Université d'Abomey-Calavi (UAC), 01 BP 526, Cotonou, République du Bénin \\ ${ }^{2}$ Access Agriculture et Université Nationale d'Agriculture, République du Bénin, 041 BP13, Cotonou, République du Bénin
}

\begin{abstract}
Résumé - Cet article se veut une contribution à la compréhension des mécanismes d'apprentissage par les vidéos en vulgarisation agricole. Trois indicateurs, le nombre de messages retenus, le nombre de messages compris et le nombre de messages appliqués, ont été utilisés pour comparer l'efficacité de trois méthodes de diffusion des informations techniques rizicoles. Il s'agit 1) de la simple mise à disposition de DVD, 2) de la projection sans facilitation et 3) de la projection avec facilitation. Un guide d'entretien a permis d'échanger avec les responsables de groupements rizicoles et les services d'encadrement tandis qu'un questionnaire a permis de collecter des données auprès de cent producteurs de riz dans trois communes du Sud du Bénin : Comé, Lokossa et Athiémé. Les résultats montrent que la simple mise à disposition de DVD permet une meilleure mémorisation et compréhension des messages contenus dans les vidéos que les deux autres méthodes. Cela montre que la présence d'un vulgarisateur/facilitateur n'est pas toujours indispensable à l'apprentissage paysan lorsque le support est adéquat et que le contenu présente un intérêt pour les producteurs. Des voies d'apprentissage non conventionnelles et peu onéreuses existent donc pour les paysans. Elles puisent leur essence dans les motivations individuelles paysannes. Ces résultats pourront aider les structures de vulgarisation agricole à choisir des méthodes de diffusion des informations par des vidéos.
\end{abstract}

Mots clés : information agricole / vidéos de formation / vulgarisation / riz / République du Bénin

\begin{abstract}
Comparing the effectiveness of three methods of dissemination of rice video-based information in Southern Benin. This paper contributes to a better understanding of video-mediated learning in extension. We used for this study three major indicators to study the effectiveness of learning after watching a video: the number of messages retained, the number of messages understood and the number of messages applied. We then compared three methods of dissemination of messages contained in videos. These are: 1) Simple DVD handing to farmers (known as handoff experiment), 2) Video projection without facilitation, and 3) Video projection with facilitation. We used a checklist to collect data from leaders of rice farmers' organisations and extension services, and a questionnaire to collect data from 100 rice farmers in three municipalities of southern Benin, namely Comé, Lokossa and Athiémé. We found that the simple DVD handing to farmers allowed a better retention and understanding of messages contained in the video compared to the other two methods. This finding shows that extension agents are not always important for farmers' learning especially when knowledge is relevant to farmers and adequately packaged. The study concludes that non-conventional ways of learning exist. They use farmers' motivation. They constitute additional ways the formal extension services could use to sustain their impact on farmers.
\end{abstract}

Keywords: agricultural information / training videos / extension / rice / Benin Republic

\footnotetext{
*Auteur correspondant : okryflorent@gmail.com
} 


\section{Introduction}

Plusieurs méthodes sont utilisées par les agents de vulgarisation pour aider les producteurs agricoles à prendre leurs décisions. Le choix de méthode que fait un agent de vulgarisation dépend de ses objectifs spécifiques, des ressources disponibles et des circonstances dans lesquelles il travaille (Van den Ban et al., 1994). Mais avec la réduction du personnel d'encadrement paysan dans la plupart des pays en développement (par exemple, au Bénin le ratio vulgarisateur/ paysan est estimé à 1/500 et, en Ouganda 1/3189 [Danielsen et al., 2015], les services de vulgarisation agricole peinent à assumer leurs fonctions avec les méthodes traditionnelles. En conséquence l'efficacité technique des producteurs baisse du fait même du manque et de l'inadéquation de leur formation technique (Kpenavoun et al., 2017). Ces dernières années, les vidéos se sont révélées de puissants outils pour atteindre plusieurs producteurs en un temps relativement court (Kouévi et al., 2014).

Au Bénin, des vidéos de formation « paysan à paysan » ont été développées suivant l'approche dite «Zooming-In, Zooming-Out» (Van Mele, 2006). Cette approche de production et de diffusion des connaissances s'appuie sur une explication approfondie des principes scientifiques qui sous-tendent une technologie ou une bonne pratique locale. Ces vidéos sont présentées par un narrateur dans un style facile à comprendre et souvent en langues locales; les démonstrations y sont faites par des producteurs expérimentés. Cela permet aux producteurs visualisant les vidéos de s'identifier aux paysans présentant la technologie et suscite ainsi en eux l'envie d'expérimenter les connaissances diffusées.

Plusieurs méthodes de diffusion des vidéos existent: la projection en se servant de vidéo-projecteurs ou de téléviseurs, la diffusion par les chaînes de télévision (Woodard, 2012) et la distribution directe de DVD (Okry et al., 2014). Les projections peuvent se faire avec ou sans facilitation. Cependant, étant donné que chaque agriculteur pourrait réagir différemment à l'information contenue dans la vidéo, la présence d'un facilitateur pourrait permettre de réajuster l'information et donc d'améliorer la compréhension (Woodard, 2012). Pour certains auteurs, la facilitation n'est pas indispensable durant les projections (Van Mele, 2011 ; Zoundji et al., 2016) d'autant que l'auditoire trouve souvent les vidéos plus convaincantes que les conseils donnés par les agents de vulgarisation (Hilou, 2012; Bentley et al., 2014a).

Au Bénin, la méthode de simple mise en place (SMEP), la méthode de projection sans facilitation (PSF), et la méthode de projection avec facilitation (PAF) ont été les plus utilisées. La méthode de simple mise en place (SMEP) a été utilisée en 2008 dans un contexte d'évaluation de la capacité des producteurs à s'auto-former et ce, sans appui extérieur (ONG, projet, service de vulgarisation agricole, etc.). La méthode de projection sans facilitation (PSF) a débuté en mars 2008 avec le contrat signé entre AfricaRice et le Cinéma numérique ambulant (CNABénin) pour la diffusion grand public de vidéos de formation en riziculture dans plusieurs villages du Bénin. Les projections couvraient aussi bien des villages rizicoles que des villages non-rizicoles mais à fort potentiel rizicole. La participation aux projections était ouverte à tout producteur désireux d'y prendre part. Il n'y avait pas de facilitateur lors de ces projections. Une animatrice collectait les questions et les impressions des participants. Enfin, la méthode de projection avec facilitation (PAF) a été utilisée dans un contexte de renforcement de la sécurité alimentaire. Des formations ont été organisées en 2009 par le ministère de l'Agriculture, de l'élevage et de la pêche (MAEP) à l'intention des riziculteurs en s'appuyant sur des vidéos. Ces formations impliquaient les responsables des groupements rizicoles qui étaient censés en faire la restitution aux autres membres. Lors de ces formations, les formateurs/ facilitateurs, pour la plupart des agents des services publics de vulgarisation, animaient un débat au terme de chaque projection.

Différents travaux, effectués sur ces trois méthodes prises individuellement, ont montré leur efficacité (Zossou et al., 2009; Okry et al., 2014). La présente étude a pour objectif de comparer l'efficacité relative de ces trois méthodes.

\section{Orientation théorique et méthodologique}

\subsection{Taxonomie de Bloom}

Nous avons orienté la conception, la collecte et l'analyse des données de cette étude en suivant la taxonomie des processus d'apprentissage de Bloom et al. (1956). Il s'agit d'une taxonomie fortement ancrée dans l'approche transactionnelle ou instructive d'apprentissage (Knight et al., 2014). En effet, c'est une représentation hiérarchisée du degré d'apprentissage de l'apprenant dans un domaine précis de la connaissance (Howard et al., 1996). Bloom et al. (1956) distinguent six classes qui caractérisent tout processus d'apprentissage: la connaissance, la compréhension, l'application, l'analyse, la synthèse et l'évaluation. La connaissance est le souvenir d'une notion antérieurement apprise. La compréhension est la capacité de l'apprenant à saisir le sens des notions reçues. L'application est l'utilisation réelle des notions reçues. L'analyse est une étape où l'apprenant déconstruit les notions reçues en leurs composantes, de façon à en analyser les relations. En phase de synthèse, l'apprenant reconstitue les composantes des notions reçues pour élaborer de nouvelles connaissances. La dernière phase est celle de l'évaluation, au cours de laquelle l'apprenant apprécie la valeur de la nouvelle connaissance qu'il a générée dans un contexte bien précis.

Mais pour Hirst (1974), cette classification perd de vue un large domaine de la "compréhension» en tant qu'objectif ultime du processus d'éducation; il pense que la classification de Bloom tend à traiter les objectifs de l'apprentissage en composantes isolées et, ce faisant, ne prend pas en compte les interactions entre les différents stades de l'apprentissage.

Pour améliorer la classification de Bloom, Anderson et Krathwohl (2001) proposent une modification de terminologie, de structure et de focus à la classification initiale. Ils ajoutent à la dimension cognitive de l'apprentissage, la dimension «processus d'apprentissage» et proposent la succession d'étapes: «se Souvenir», «Comprendre», «Appliquer», «Analyser», «Évaluer» et «Créer».

Ces deux dimensions forment les deux piliers essentiels de la vulgarisation agricole: la connaissance apportée aux producteurs entre dans un processus d'apprentissage et conduit à un nouvel état de connaissance. Nous avons utilisé la classification de Bloom mais, vu nos contraintes de temps et de budget, nous avons limité notre analyse aux trois premières 
Tableau 1. Description des vidéos mises en place et diffusées dans le milieu d'étude. Vidéos disponibles sur www.accessagriculture.org. Table 1. Description of the videos disseminated in the study area.

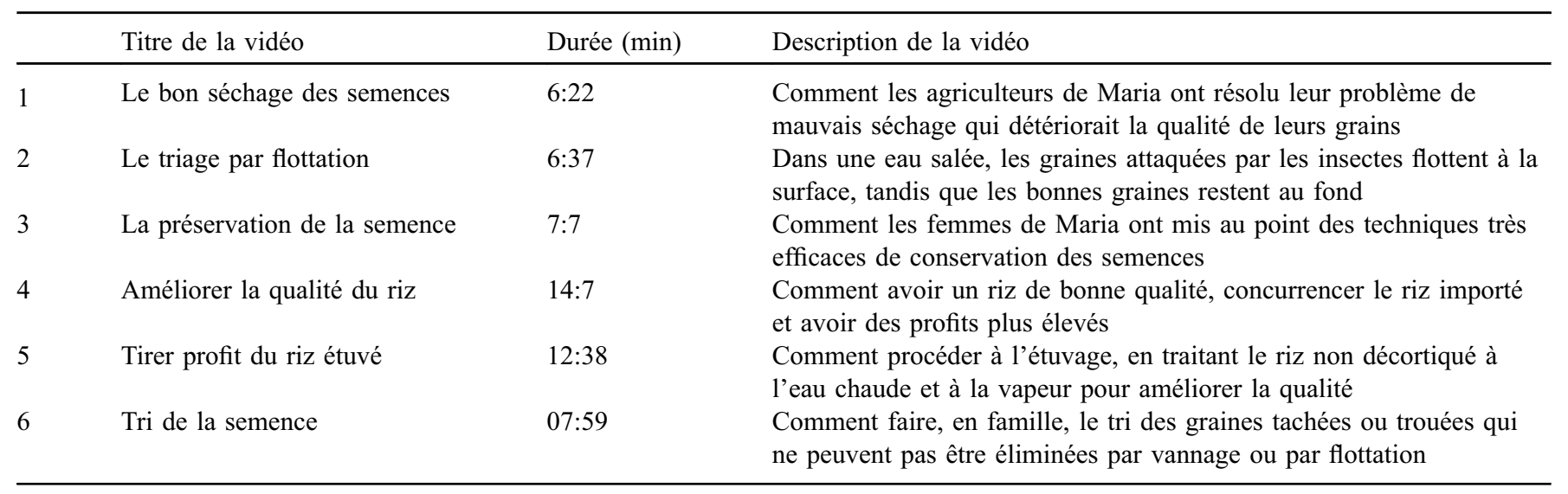

étapes : le souvenir, la compréhension et de l'application des messages diffusés à l'aide des supports vidéos.

\section{Méthodologie}

\subsection{Sites d'étude et échantillonnage}

La présente étude s'est déroulée de septembre 2013 à mars 2014 dans le département du Mono, au Sud-Ouest du Bénin, dans les communes de Comé, d'Athiémé et de Lokossa. La collecte de données a été réalisée dans six villages, à raison de deux villages par méthode de dissémination étudiée. Ces villages ont été choisis suite à une revue de littérature sur l'historique de l'utilisation des vidéos et après une phase exploratoire. Les villages de Gatchivè et d'Adamè ont testé la simple mise en place des vidéos (SMEP), les villages d'Ayoukomey et d'Aligoudo ont bénéficié de la projection sans facilitateur (PSF), et les villages de Hlodo et Tinou ont bénéficié de la projection avec facilitateur (PAF). Le tableau 1 présente les vidéos de cette étude.

$\mathrm{Au}$ total, cent producteurs ont été interrogés. Tous ont bénéficié du contenu des vidéos entre 2008 et 2009. Trente producteurs ont été interrogés dans les villages de Gatchivè et d'Adamè ayant bénéficié de la SMEP, et trente dans les villages de Hlodo et Tinou ayant bénéficié de la PAF. Quarante producteurs ont été interrogés à Ayoukomey et Aligoudo, bénéficiaires de la PSF, car cette méthode a touché plus de producteurs. L'effet du temps écoulé entre les projections/mise en place des vidéos et la réalisation de l'enquête est le même pour tous les producteurs : cinq à six ans. Ce temps important est susceptible de réduire la capacité des producteurs à se souvenir des messages véhiculés par les vidéos.

Dans chacun des villages nous avons procédé:

- à l'identification des bénéficiaires de DVDs et des participants aux projections, en nous servant des listes lorsque celles-ci existaient. Pour les villages PSF où la liste des participants n'était pas disponible, nous avons procédé à un sondage pour identifier les premiers participants aux projections ;
- à la constitution de listes plus ou moins exhaustives des bénéficiaires de vidéos et participants aux projections à partir des listes issues du sondage (pour la PSF);

- à la sélection aléatoire des interviewés.

\subsection{Collecte des données}

En octobre 2013 (phase exploratoire), un guide d'entretien a permis de collecter des premières informations auprès d'une quinzaine de producteurs, des agents des services d'encadrement rural des trois communes d'étude, du Groupe d'action pour un développement durable (GAAD), une ONG impliquée dans l'apprentissage axé sur les vidéos, et des organisations de producteurs. Les discussions avec ces personnes ressources ont porté sur les interventions en faveur du développement de la riziculture, le contenu des six vidéos utilisées, la pertinence pour les producteurs des messages contenus dans les vidéos, et le contexte d'utilisation de ces vidéos.

Les six vidéos identifiées ont été regardées attentivement par trois personnes différentes, qui ont inventorié tous les messages qu'elles contiennent. La synthèse des travaux de ces trois personnes a servi de base à l'élaboration du questionnaire d'enquête. Ce dernier portait sur le nombre de messages techniques retenus, compris et appliqués par les producteurs, les pratiques culturales avant et après la diffusion ou l'utilisation des vidéos et les caractéristiques sociales des producteurs interrogés, notamment le sexe, le niveau d'instruction, les activités génératrices de revenus, etc.

Enfin, en utilisant l'observation directe, nous avons pu rapprocher les déclarations des producteurs sur la mise en application des messages techniques appris avec leurs pratiques réelles. Nous avons pour cela visité les champs ou les unités de production des riziculteurs ayant déclaré qu'ils ont appliqué ou appliquent encore des techniques enseignées dans les vidéos.

\subsection{Analyse des données}

L'analyse des données a été faite sur la base de trois indicateurs inspirés des travaux de Bloom et al. (1956): messages retenus, messages compris et messages appliqués. 
- Pourcentage des messages retenus (PMR)

$$
\operatorname{PMR}(\%)=\frac{\text { Nombre de messages retenus }}{\begin{array}{l}
\text { Nombre total de messages } \\
\text { inventoriés dans les vidéos }
\end{array}} \times 100
$$

- Pourcentage des messages compris (PMC)

$\operatorname{PMC}(\%)=\frac{\begin{array}{l}\text { Nombre de messages retenus }- \\ \text { Nombre messages modifiés }(\text { mal exprimés })\end{array}}{\begin{array}{c}\text { Nombre total de messages } \\ \text { inventoriés dans les vidéos }\end{array}} \times 100$

- Pourcentage des messages appliqués au moins une fois (PMA)

$$
\operatorname{PMA}(\%)=\frac{\begin{array}{c}
\text { Nombre de messages } \\
\text { appliqués au moins une fois }
\end{array}}{\begin{array}{c}
\text { Nombre total de messages } \\
\text { inventoriés dans les vidéos }
\end{array}} \times 100
$$

L'efficacité des méthodes par rapport au nombre de messages retenus, compris et appliqués a été analysée dans un premier temps par une analyse de la variance qui a permis de faire ressortir la méthode efficace et dans un second temps en utilisant les modèles de régression de Poisson, de quasiPoisson, binomial négatif, de Poisson avec inflation de zéro et binomial négatif avec inflation de zéro, afin de faire ressortir les différences éventuelles d'efficacité entre les messages selon la méthode de diffusion. Le critère d'information d'Akaike (AIC) a été utilisé pour choisir le meilleur modèle. Pour les trois indicateurs, le modèle binomial négatif avec inflation de zéro a été retenu dans cette étude.

\section{Résultats et analyses}

\subsection{Caractéristiques sociales des producteurs et effets des vidéos}

Le tableau 2 montre que les producteurs interrogés, en plus des activités agricoles qui constituent leurs activités principales, des activités secondaires génératrices de revenus. Pour les producteurs soumis à la PSF, la production du riz n'apparaît pas comme une activité principale, car au moment de nos enquêtes (2013-2014), les producteurs ne cultivaient plus de riz. Cela est essentiellement dû au fait que les participants aux PSF n'étaient pas sélectionnés suivant un critère précis. Les projections étaient ouvertes à tout producteur désireux d'y prendre part. Le CNA démarrait les projections avec de la musique pour attirer les producteurs. L'intérêt que ceux-ci portaient à l'évènement n'était donc pas nécessairement un intérêt pour la production rizicole.

Peu de producteurs possèdent des lecteurs de DVD, entre $10 \%$ et $25 \%$. Cela n'a pas empêché certains producteurs concernés par la PAF et la SMEP de visualiser plusieurs fois les vidéos. Ce qui n'a pas été le cas des producteurs concernés par la PSF, puisqu'ils n'avaient pas accès aux DVD. Malgré ces freins à la visualisation répétée (qui pourrait améliorer l'apprentissage), on note que toutes les méthodes ont apporté des changements dans les pratiques paysannes. Par exemple, après la visualisation des vidéos rizicoles (PSF) dans des villages non-rizicoles, certains producteurs se sont lancés dans la riziculture. Mais faute d'accompagnement technique et de moyens, à en croire nos informateurs, ces producteurs ont dû abandonner la riziculture

Tableau 2. Caractéristiques sociales des producteurs.

Table 2. Social characteristics of the studied farmers.

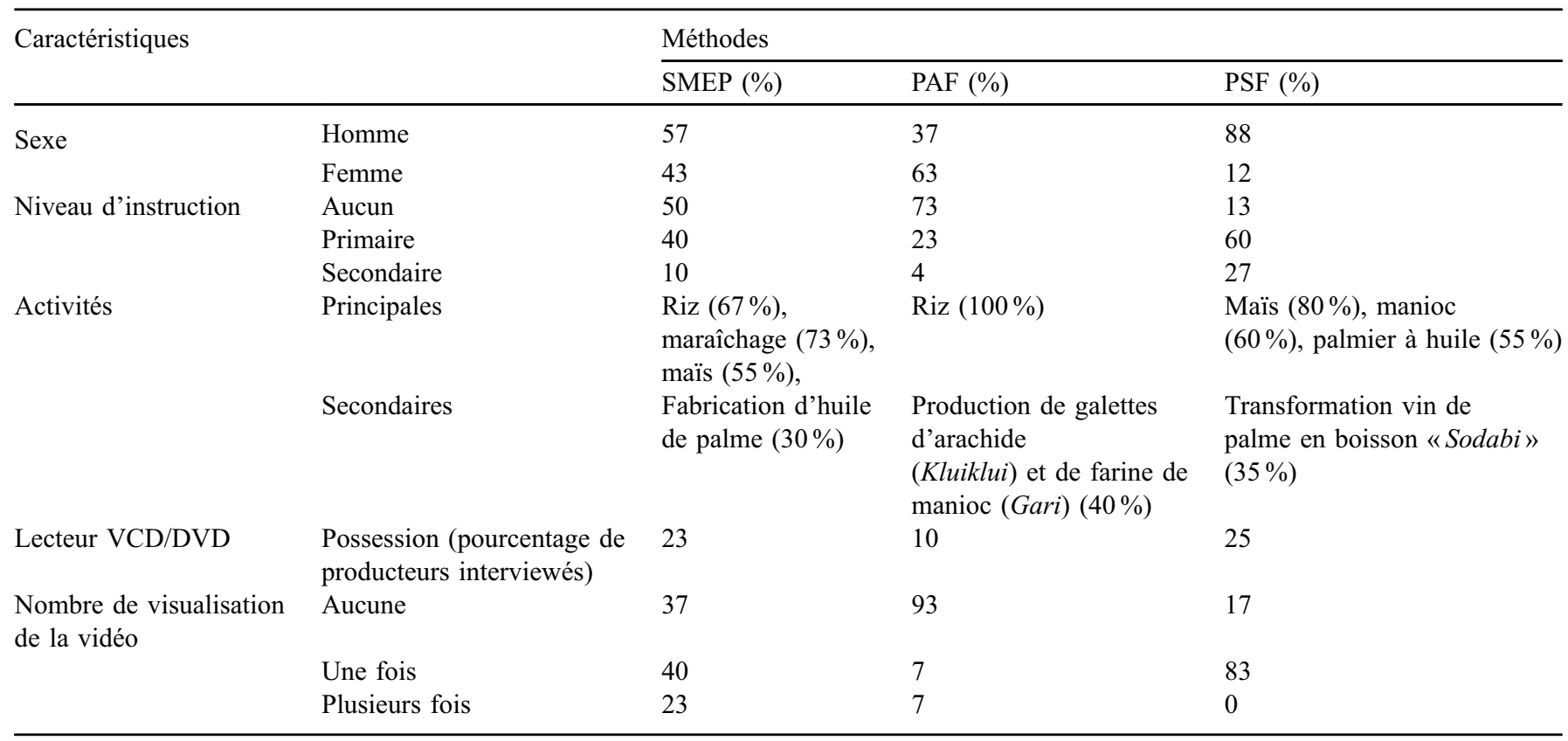

SMEP : simple mise en place; PAF : projection avec facilitateur; PSF : projection sans facilitateur. 
Tableau 3. Répartition des producteurs (pourcentage) par méthode de diffusion selon le niveau (pourcentage) de messages retenus, compris et appliqués.

Table 3. Repartition of producers per method of dissemination following the level (percentage) of messages retained, understood and applied.

\begin{tabular}{|c|c|c|c|c|c|c|}
\hline & & \multicolumn{5}{|c|}{ Pourcentage de messages } \\
\hline \multirow[t]{3}{*}{ Messages retenus par les producteurs (\%) } & SMEP & 37 & 27 & 30 & 7 & 0 \\
\hline & PSF & 93 & 8 & 0 & 0 & 0 \\
\hline & PAF & 87 & 7 & 7 & 0 & 0 \\
\hline Messages compris par les producteurs (\%) & SMEP & 37 & 27 & 30 & 7 & 0 \\
\hline \multirow[t]{3}{*}{ Messages appliqués par les producteurs (\%) } & SMEP & 90 & 7 & 3 & 0 & 0 \\
\hline & PSF & 90 & 10 & 0 & 0 & 0 \\
\hline & PAF & 94 & 3 & 3 & 0 & 0 \\
\hline
\end{tabular}

SMEP : simple mise en place; PAF : projection avec facilitateur; PSF : projection sans facilitateur.

au profit de leurs anciennes activités agricoles. Pour les deux autres méthodes, la visualisation des vidéos aurait permis une large adoption du semis en ligne.

La comparaison se fait donc entre des villages et des producteurs qui ne présentent pas exactement les mêmes caractéristiques, ce qui pourrait introduire un biais.

\subsection{Comparaison de l'efficacité des trois méthodes sur la base du nombre de messages retenus par les producteurs}

Le tableau 3 montre que la majorité des producteurs ont un faible niveau de mémorisation des messages (entre $0 \%$ et $20 \%$ ), que ce soit avec la PSF ou la PAF. Cela serait dû à l'abandon de la riziculture par certains producteurs et au faible niveau d'instruction chez d'autres (Tab. 2). On voit que la SMEP permet à de plus nombreux producteurs de retenir plus de messages (jusqu'à environ $80 \%$ des messages). L'analyse de variance réalisée sur les pourcentages moyens de messages retenus par méthode révèle aussi que la SMEP est la plus efficace (différence statistiquement significative: $\mathrm{F}=25,91$; $\mathrm{dl}=2 ; p<0,001)$. En outre, la régression binomiale négative avec inflation de zéro réalisée sur les trois méthodes (en prenant la PAF comme référence) révèle pour la PSF un coefficient négatif, une probabilité non significative et un ratio inférieur à $1(\mathrm{Coef}=-0,10 ; p>0,05 ; \mathrm{OR}=0,91)$ et pour la SMEP un coefficient positif, une probabilité significative et un ratio supérieur à $1(\mathrm{Coef}=1,33 ; p<0,01 ; \mathrm{OR}=3,79)$. La SMEP permet donc de retenir, de façon significative, environ 4 fois plus de message que la PAF.

\subsection{Comparaison de l'efficacité des trois méthodes sur la base du niveau de compréhension des messages}

Le tableau 3 montre que la majorité des producteurs ont un faible niveau de compréhension des messages (entre $0 \%$ et $20 \%$ ) avec la PSF et la PAF. Par contre, la SMEP permet à un plus grand nombre de producteurs de comprendre plus de messages (jusqu'à environ $80 \%$ des messages). L'analyse de variance réalisée sur les pourcentages moyens de messages compris par méthode révèle aussi que la SMEP est plus efficace (différence statistiquement significative: $\mathrm{F}=22,67$; $\mathrm{dl}=2 ; p<0,001)$.

La régression binomiale négative avec inflation de zéro réalisée sur les trois méthodes (en prenant la PAF comme référence) révèle pour la PSF un coefficient négatif, une probabilité non significative et un ratio inférieur à 1 (Coef $=-$ $0,08 ; p>0,05 ; \mathrm{OR}=0,92)$ et pour la SMEP un coefficient positif, une probabilité significative et un ratio supérieur à 1 $($ Coef $=1,27 ; p<0,01 ; \mathrm{OR}=3,57)$. La SMEP permet donc de comprendre environ 4 fois plus de messages que la PAF.

\subsection{Comparaison de l'efficacité des trois méthodes sur la base du nombre de messages appliqués}

Le taux d'application des messages varie de $0 \%$ à $20 \%$ pour les trois méthodes (Tab. 3). Les producteurs des villages avec PSF, après avoir visualisé les vidéos, s'étaient lancés dans la production rizicole en appliquant les messages contenus dans les vidéos. Mais les difficultés afférentes à la riziculture et à l'approvisionnement en matières premières les ont découragés. L'efficacité relative de la PSF n'a donc pas été durable et n'a pas résisté à l'expérimentation concrète.

Certains producteurs expliquent la non-application par le fait qu'ils possédaient déjà certains équipements promus dans les vidéos. C'est le cas par exemple des producteurs qui disposaient déjà d'aires de séchage et de petites bâches et n'ont donc pas eu à appliquer la technique présentée dans la vidéo de séchage. À l'inverse, certaines productrices interrogées restent attachées à leurs pratiques de production rizicole et ne sentent pas la nécessité de les modifier. En outre, la mauvaise compréhension du contenu de certaines vidéos peut diminuer la motivation à les adopter. C'est le cas par exemple du paysan Jean qui a visualisé la vidéo «Triage par flottation » et qui ne l'a pas adoptée parce qu'il l'a mal comprise. Cette vidéo montre comment les graines de riz de mauvaise qualité et attaquées par les insectes flottent à la surface de l'eau. Mais Jean, lui, a compris que: «la technique de tri par flottation présentée dans la vidéo devrait déclencher la germination or le 
test de germination est fait chez nous avant l'achat de la semence. Nous n'avons plus besoin de le faire à nouveau. C'est la raison pour laquelle moi je ne le fais pas ».

Les pourcentages moyens d'application des messages par méthode ne sont pas significativement différents.

\section{Discussion}

Plusieurs raisons expliquent l'efficacité en termes de mémorisation et de compréhension des messages contenus dans les vidéos diffusées par simple mise en place (SMEP) de DVD. Les principales sont énumérées ci-dessous.

\subsection{La SMEP permet de voir et de revoir les vidéos}

La SMEP offre la possibilité aux producteurs de voir et de revoir les vidéos car les producteurs SMEP avaient eu accès aux copies des DVD. En effet, tous les producteurs qui ont retenu de $50 \%$ à $70 \%$ des messages contenus dans les vidéos, ont visualisé ces vidéos en moyenne trois fois et ont aussi une compréhension des messages retenus allant de $40 \%$ à $65 \%$. En visualisant à plusieurs reprises les vidéos, les producteurs s'approprient mieux les messages et leur niveau de compréhension s'améliore. La visualisation à plusieurs reprises des vidéos constitue un facteur important dans le processus d'apprentissage. Cela rejoint Bédé (2016), dont les travaux dans une région voisine ont montré que la répétition double, voire triple, le nombre de messages vidéo retenus et compris par les producteurs. À la suite de Bentley et al. (2014b) qui ont conduit une étude similaire au Mali, nous recommandons donc que les appuis à la vulgarisation agricole par les vidéos se fasse sous forme de mise à disposition des vidéos auprès des communautés concernées, pour permettre une répétition des projections.

\subsection{La SMEP permet de visualiser la vidéo au moment désiré}

De par sa nature, la SMEP offre la possibilité aux producteurs de visualiser les vidéos quand ils le désirent. Cela constitue un facteur important, dans la mesure où tous les moments ne sont pas propices à l'apprentissage (Karubanga et al., 2016a). Pour ces producteurs, le moment opportun pour regarder ces vidéos est à la veille d'une nouvelle campagne rizicole. À ce moment-là, les producteurs sont plus attentifs. Leur motivation constitue un élément important pour bien suivre les vidéos et par conséquent mieux conserver et mieux comprendre les messages. C'est ce que stipule l'approche subjectiviste ou affectiviste de l'apprentissage, qui considère que l'on apprend quand on le désire. Elle recherche donc les raisons de l'apprentissage dans les motivations de l'apprenant (Bruner, 1982; Ratner, 2008). Selon Chiouse (2001), la motivation à apprendre constitue un facteur important pour un apprentissage réussi : aimer apprendre et vouloir apprendre passent par la découverte des satisfactions qui peuvent résulter d'un apprentissage réussi. Ruph (2010) le rejoint: «La motivation à l'apprentissage, la valeur affective et les émotions jouent un rôle capital dans l'enregistrement, la mémorisation et la récupération des connaissances. Une motivation intrinsèque, le plaisir d'apprendre, l'intérêt manifesté pour le sujet, la signification profonde de cet apprentissage, ont les meilleurs effets ». Et c'est justement ce à quoi on aboutit auprès des producteurs quand la projection est faite au moment voulu par ces derniers. La possibilité de visualiser des vidéos au bon moment qu'offre la SMEP a donc probablement contribué à la rendre plus efficace que les autres méthodes en termes de messages retenus et compris.

\subsection{La méthode de SMEP permet le co-apprentissage entre producteurs}

Pendant les visualisations, d'intenses échanges se font entre les producteurs dans le but de mieux comprendre les vidéos (Karubanga et al., 2016b). Les producteurs qui organisent des projections répétées comprennent mieux et facilitent le processus d'apprentissage des autres. Tout cela amène à un renforcement des relations entre producteurs. Lie et Mandler (2009) rapportent que, de plus en plus, les producteurs s'organisent pour visionner des vidéos pertinentes pour leurs activités professionnelles. Cela est confirmé dans les villages de SMEP (Adamè et Gatchivè), où les producteurs se sont organisés pour visualiser les vidéos. Après visualisation, des groupes de producteurs ont vu le jour dans le but de mutualiser leurs efforts. La SMEP permet donc le renforcement des relations entre les producteurs. Dans ce processus social de co-apprentissage, les messages contenus dans les vidéos sont partagés entre producteurs, ce qui augmente leur capacité de mémorisation et leur niveau de compréhension.

\section{Conclusion}

Cette comparaison de trois méthodes de diffusion de l'information contenue dans les vidéos, montre que la méthode de simple mise en place est la plus efficace en termes de mémorisation et de compréhension des messages contenus dans les vidéos. Cette efficacité lui est conférée par la possibilité qu'ont les producteurs de visualiser à plusieurs reprises, et quand ils le veulent, les vidéos qui leur ont été données. Le co-apprentissage social qui en résulte renforce la compréhension des connaissances véhiculées par les vidéos. Malgré les probables biais dus au fait que les villages n'ont pas les mêmes caractéristiques et ne sont pas identiques par leur histoire rizicole, les résultats de cette étude suggèrent que les efforts de vulgarisation s'appuyant sur l'utilisation des vidéos devraient privilégier la mise à disposition des vidéos, pour favoriser les visualisations répétées. Les récents développements technologiques ont conduit des spécialistes de l'apprentissage par les vidéos comme Access Agriculture (www.accessagriculture.org) à convertir plus de 1000 vidéos de formation en version $3 \mathrm{GP}$, compatible avec les téléphones cellulaires ordinaires. Cela offre de nouvelles opportunités aux services de vulgarisation et de conseil agricole pour fournir aux producteurs des vidéos de formation à visualiser quand ils le désirent.

Remerciements. Nous remercions Access Agriculture pour avoir soutenu la présente recherche par le projet «Videos for Farmers » financé par la Direction du Développement et de la Coopération (Suisse) [Project Numéro 7F-08378.01]. Nous 
remercions aussi les évaluateurs anonymes et tous ceux qui ont contribué à améliorer cet article.

\section{Références}

Anderson LW, Krathwohl DR. 2001. A taxonomy for learning, teaching and assessing: A revision of Bloom's Taxonomy of educational objectives. New York: Longman, $68 \mathrm{p}$.

Bentley J, Van Mele P, Okry F., Zossou E. 2014a. Videos that speak for themselves: when non-extensionists show agricultural videos to large audiences. Development in Practice 24(7): 921-929. DOI: 10.1080/09614524.2014.942216.

Bentley J, Van Mele P, Zoundji G, Guindo S. 2014b. Social innovations triggered by videos: Evidence from Mali. Agro-Insight Report for Access Agriculture, 27 p. [2017/10/27]. http:// agroinsight.com/downloads/Articles-Agricultural-Extension/ 2014 Social-innovations-triggered-by-videos-in-Mali-Bentley-etal-2014.pdf.

Bloom BS, Englehart, MD, Furst EJ, Hill WH, Krathwohl DR. 1956. Taxonomy of educational objectives. Handbook 1: Cognitive domain. New York: Longmans, Green.

Bruner J. 1982. The language of education. Social Research 49(4): 835-853.

Chiouse S. 2001. Examen thématique de l'apprentissage des adultes. «Pédagogie et apprentissages des adultes. État des lieux et recommandations ». Document de travail. OCDE, 141 p. [2017/10/27]. https://hal.archives-ouvertes.fr/hal-00133764/document.

Danielsen S, Karubanga G, Mulema J. 2015. Institutionalization. Listening to the silent patient. Uganda's journey towards institutionalizing inclusive plant health services. CABI working paper 7: 37-55. DOI: 10.1079/CABIPLANT.

Hilou K. 2012. Rapport d'évaluation de l'utilisation de la vidéo pour la formation des adhérents au service de conseil à l'exploitation familiale (CEF) de l'UGCPA/BM. Ouagadougou, Burkina Faso : l'Université d'Ouagadougou, 78 p. [2017/10/27]. https://www.fondation-farm.org/ IMG/pdf/Rapport_video_FARM_UGCPA_mai12.pdf.

Hirst PH. 1974. Knowledge and curriculum: A collection of philosophical papers. London, Boston: Routledge and Kegan Paul, 193 p.

Howard RA, Carver CA, Lane WD. 1996. Felder's learning styles, Bloom's taxonomy, and the Kolb learning cycle: tying it all together in the CS2 course. SIGCSE '9S 2/S6 ACM O-S9791-757 -IU9WOO02. [2017/10/27]. https://www.researchgate.net/pro file/Curtis_Carver/publication/221538553_Felder\%27s_Lear ning_Styles_Bloom\%27s_Taxonomy_and_the_Kolb_Learning Cycle_Tying_it_All_Together_in_the_CS2_Course/links/ 0fcfd50 d0cb9bb6e-89000000/Felders-Learning-Styles-BloomsTaxonomy-and-the-Kolb-Learning-Cycle-Tying-it-All-Togetherin-the-CS2-Course.pdf.

Karubanga G, Kibwika P, Okry F, Sseguya H. 2016a. How the timing and location of video shows influence learning among rice farmers in Uganda? Int J Agric Res Innov \& Tech 6(2): 77-81.

Karubanga G, Kibwika P, Okry F, Sseguya H. 2016b. Empowering farmers to learn and innovate through integration of video- mediated and face-to-face extension approaches: The case of rice farmers in Uganda. Cogent Food and Agriculture 2(1): 1-12 p. DOI: $10.1080 / 23311932.2016 .1274944$.

Knight S, Buckingham SS, Littleton K. 2014. Epistemology, Assessment, Pedagogy: Where Learning Meets Analytics in the Middle Space. Journal of Learning Analytics 1(2): 23-47. DOI: 10.18608/jla.2014.12.3.

Kouévi A, Okry F, Van Mele P, Muchina B, Rodgers J et Malone P. 2014. Improving livelihoods of rural people through quality training videos: Lessons from Access Agriculture. Paper presented at the Tropentag Conference on International Research on Food Security, Natural Resource, Management and Rural Development. Prague, Czech: Czech University of Life Sciences. [2017/10/27]. https://www.accessagriculture.org/sites/default/files/upload/files/ Improving\%20Livelihoods\%20through\%20Quality\%20Training \%20Videos\%20-\%20Kouevi\%20et\%20al\%202014.pdf.

Kpenavoun CS, Gandonou E, Fiogbe N. 2017. Mesure de l'efficacité technique des petits producteurs d'ananas au Bénin. Cahiers Agricultures 26: 25004. DOI: 10.1051/cagri/2017008.

Lie R, Mandler A. 2009. La vidéo dans le développement: Filmer pour le changement rural. Wageningen, The Netherlands: CTA, $399 \mathrm{p}$.

Okry F, Van Mele P, Houinsou F. 2014. Forging new partnerships: lessons from the dissemination of agricultural training videos in Benin. The Journal of Agricultural Education and Extension 20(1): 27-47. DOI: 10.1080/1389224X.2013.783495.

Ratner C. 2008. Cultural psychology and qualitative methodology: Scientific and political considerations. Culture and Psychology 14(3): 259-288. DOI: 10.1177/1354067X08088557.

Ruph F. 2010. Comment mieux mémoriser. [2015/06/09]. http:// web2.uqat.ca/guidestrategies/memoire/savoirplus.php.

Van den Ban A, Hawkins H, Brouwers J, Boon C. 1994. La vulgarisation rurale en Afrique. Les méthodes de vulgarisation. Ed. Karthala et CTA, 144 p.

Van Mele P. 2006. Zooming-in, zooming-out: a novel method to scale up local innovations and sustainable technologies. International Journal of Agricultural Sustainability 4(2), 131-142.

Van Mele P. 2011. Video-mediated farmer-to-farmer learning for sustainable agriculture. A scoping study for SDC, SAI Platform and GFRAS. Ghent, Agro-Insight Report, 47 p. [2017/10/27]. http://agroinsight.com/downloads/articles-divers/Farmer-to-far mer-video-FINALREPORT-Van-Mele-2011.pdf.

Woodard J. 2012. Intégrer la vidéo à moindre coût dans les projets de développement agricole : un kit destiné aux experts. FHI 360, Avril 2012, 228 p. [2017/10/27]. http://ictforag.org/toolkits/video/down loads/LowCostVideoToolkit-French.pdf.

Zossou E, Van Mele P, Vodouhe SD, Wanvoeke J. 2009. Comparing farmer-to-farmer video with workshops to train rural women in improved rice paroling in Central Benin. The Journal of Agricultural Education and Extension 15(4): 329-339.

Zoundji G, Okry F, Vodouhê S, Bentley J. 2016. The distribution of farmer learning videos: Lessons from non-conventional dissemination networks in Benin. Cogent Food and agriculture 2: 1-15. DOI: 10.1080/23311932.2016.1277838.

Citation de l'article : Davito T, Okry F, Kouevi A, Vodouhe S. 2017. Efficacité comparée de trois méthodes de diffusion d'informations rizicoles par des vidéos au Sud du Bénin. Cah. Agric. 26: 65003. 